\title{
Intelligent Setting Control of Raw Meal Calcination Proces
}

\author{
Jinghui Qiao, Tianyou Chai, and Hong Wang
}

\begin{abstract}
In raw meal calcination process, the target value of decomposition ratio of raw meal (RMDR) is different in easy calcination stage and difficult calcination stage because boundary conditions of raw meal change frequently, where RMDR cannot be guaranteed within its desirable ranges. To solve this problem, an intelligent setting control method is proposed. This method for raw meal calcination process consists of five modules, namely a RMDR target value setting model using subtraction clustering method (SCM) and adaptivenetwork-based fuzzy inference system containing categorical input (C-ANFIS), a control loop pre-setting model, a feedback compensation model based on fuzzy rules, a feedforward compensation model based on fuzzy rules, and a soft measurement model for RMDR. The proposed method is realized by on-line adjusting the setpoints of control loops with the change of raw meal boundary conditions. This method has been successfully applied to the raw meal calcination process of Jiuganghongda Cement Plant in China and its efficiency has been validated by the practical application results.
\end{abstract}

\section{INTRODUCTION}

Raw meal calcination in calciner is a complex chemical reaction. Technology index, namely decomposition ratio of raw meal (RMDR), indicates the quality and the efficiency of the product processing. It is desirable to keep its required ranges. The control objective of raw meal calcination process is to obtain the largest possible RMDR in easy calcination stage or difficult calcination stage. However, the RMDR is difficult to be measure on-line. Moreover, the dynamic characteristics between the RMDR and the calciner temperature and the preheater $\mathrm{C} 1$ outlet temperature shows strong nonlinearities, and coupling effects. This relationship vary with the operation conditions. Therefore, it is difficult to obtain the RMDR by a precise mathematical model.

In actual production processes, the RMDR was measured offline once an hour[1]. The on-site operators would adjust the setpoints of control loops by using laboratory measuring results. Since boundary conditions (i.e., flow, ingredients and particle size.) of raw meal change frequently, such manual operation cannot adjust the setpoints of control loops in time, which in turn implies that the RMDR cannot be controlled within its required ranges. In this case, it would not only lead to low production capacity but also increase next procedure $\operatorname{load}[2]$.

Manuscript received March 12, 2011. This work was supported in part by the National Key Fundamental Research Program of China (No.2009CB320601), the Funds for Creative Research Groups of China(60821063), and 111 Program (B08015).

J. H. Qiao and T. Y. Chai are with the State Key Laboratory of Synthetical Automation for Process Industries and with the Research Center of Automation, Northeastern University, Shenyang 110004, China qiaojh2002@163. com

$\mathrm{H}$. Wang is with the school of Electrical and Electronic Engineering, University of Manchester, U.K. hong.wang@manchester.ac.uk
For raw meal calcination processes, most of the literature focused on equipment design and simulation. To optimize the design of the calciner, a dynamic mathematical model for an in-line low-NOx calciner using non-isothermal diffusionreaction models for char combustion and limestone calcination has been established in [3]. A simulation model for RMDR has been established by Koumboulis et al. based on coal feeder to calciner [4]. The above method on equipment design and simulation does not consider the impact of boundary conditions of raw meal, and the setpoints of the control loops are consistent.

To overcome the above shortcomings, an intelligent setting control method has been proposed in this paper. The intelligent setting control method for raw meal calcination process consists of five modules, namely a RMDR target value setting model based on SCM and C-ANFIS, a control loop pre-setting model, a feedback compensation model based on fuzzy rules, a feedforward compensation model based on fuzzy rules, and a soft measurement model for RMDR. The proposed approach can adjust the setpoints of the control loops on-line with the change of raw meal boundary conditions and control the technology index into its desirable range. This method has been successfully applied to the raw meal calcination process and its efficiency has been validated by the practical application results.

\section{DESCRIPTION OF RAW MEAL CALCINATION PROCESS}

\section{A. Description of Raw Meal Calcination Process}

The flowsheet of the raw meal calcination process with an annual production capacity of 0.73 million ton is shown in Fig.1. In the suspension preheater system with the calciner and the rotary kiln, the exhaust gas from the rotary kiln and the calciner are passed from the bottom preheater C5 to the humidifier tower (H.T.) by the high-temperature fan and the exhaust fan. The raw meal from homogenizing silo is fed into the top preheater $\mathrm{C} 1$ inlet duct for heat exchange with calciner and kiln exhaust gas, then collected separately with a cyclone and charged into the inlet duct of the next lower cyclone. Heat exchanged and collected is then repeated. The raw meal is heated sequentially with the stages of cyclones, calcined with the decomposition ratio of 0.85-0.94 (dimensionless) in calciner, then fed into the rotary kiln. The coal feeding from the coal powder scale and the auxiliary air from the auxiliary air blower are mixed into a two-phase fuel flow, sprayed into the calciner. They are then combusted with the tertiary air, which comes from the kiln head hood. 


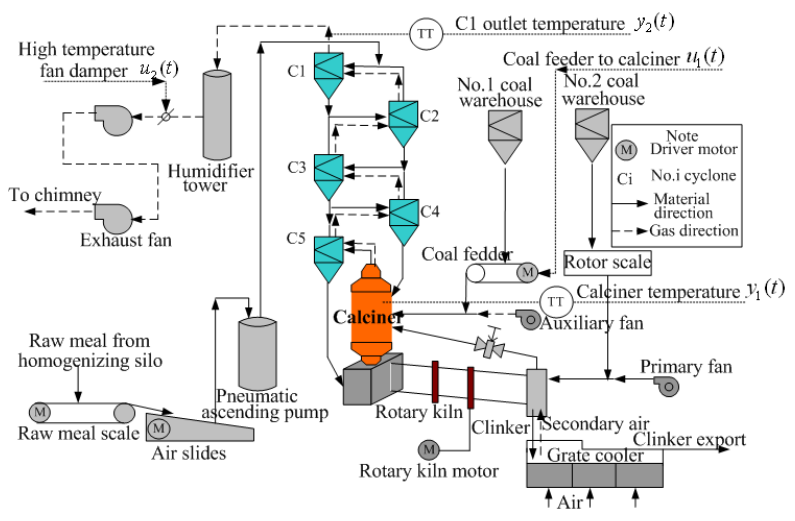

Fig. 1. Flowsheet of the raw meal calcination process

\section{B. Control Objective}

According to the physical analysis of raw meal calcination process, these boundary conditions consist iron oxide content $B_{1}$, alumina oxide content $B_{2}$, silica oxide content $B_{3}$, raw meal particle size $B_{4}$, raw meal flow $B_{5}$ and kiln tail temperature $B_{6}$, which affect RMDR [5]. In practical production process, the RMDR $\gamma(t)$ is related to the calciner temperature $y_{1}$ and preheater $\mathrm{C} 1$ outlet temperature $y_{2}$. Its dynamics can be described as follows:

$$
\gamma(t)=f\left(y_{1}, y_{2}, B_{1}, \ldots, B_{6}\right)
$$

where $f(\cdot)$ is an unknown nonlinear function varying with the boundary conditions $B=\left[B_{1}, B_{2}, B_{3}, B_{4}, B_{5}, B_{6}\right]$.

In actual production process, the content of free $\mathrm{CaO}$ (f-CaO) in samples was defined as raw meal calcination index when calcining temperature is $1450^{\circ} \mathrm{C}$ [6]. To simplify, this index denoted by $f_{c 1450}$. Condition was defined as easy calcination stage when $f_{c 1450}$ less or equal to $1.5 \%$; Condition was defined as difficult calcination stage when $f_{c 1450}$ greater than $1.5 \%$ and less or equal to $2.5 \%$. The RMDR target value in two stages is not the same. To obtain the highest possible RMDR $\gamma(t)$ in easy calcination stage $(\alpha=1)$ or difficult calcination stage $(\alpha=0)$, the control objective is described as:

$$
\begin{aligned}
& \min \left[\gamma^{*}-\gamma(t)\right] \\
& \quad \text { s.t. } \gamma(t)=f\left(y_{1}, y_{2}, B_{1}, \ldots, B_{6}\right) \\
& \quad \alpha \gamma_{L}^{e}+(1-\alpha) \gamma_{L}^{d} \leq \gamma(t) \leq \alpha \gamma_{H}^{e}+(1-\alpha) \gamma_{H}^{d} \\
& \quad \alpha y_{1 L}^{e}+(1-\alpha) y_{1 L}^{d} \leq y_{1} \leq \alpha y_{1 H}^{e}+(1-\alpha) y_{1 H}^{d} \\
& \quad \alpha y_{2 L}^{e}+(1-\alpha) y_{2 L}^{d} \leq y_{2} \leq \alpha y_{2 H}^{e}+(1-\alpha) y_{2 H}^{d},
\end{aligned}
$$

where $\gamma(t)=f\left(y_{1}, y_{2}, B_{1}, \ldots, B_{6}\right)$ is the nonlinear dynamic function, $\gamma^{*}$ is the RMDR target value, $\gamma_{L}^{e} \leq \gamma^{*} \leq$ $\gamma_{H}^{d} ; y_{1}$ is the calciner temperature, $y_{2}$ is the preheater $\mathrm{C} 1$ outlet temperature; $\gamma_{L}^{e}, y_{1 L}^{e}$ and $y_{2 L}^{e}$ are the lower limit of RMDR, calciner temperature and preheater $\mathrm{C} 1$ outlet temperature in easy calcination stage, respectively; $\gamma_{L}^{d}, y_{1 L}^{d}$ and $y_{2 L}^{d}$ are the lower limit of RMDR, calciner temperature and preheater $\mathrm{C} 1$ outlet temperature in difficult calcination stage, respectively; $\gamma_{H}^{e}, y_{1 H}^{e}$ and $y_{2 H}^{e}$ are the higher limit of RMDR, calciner temperature and preheater $\mathrm{C} 1$ outlet temperature in easy calcination stage, respectively; $\gamma_{H}^{d}, y_{1 H}^{d}$ and $y_{2 H}^{d}$ are the higher limit of RMDR, calciner temperature and preheater $\mathrm{C} 1$ outlet temperature in difficult calcination stage, respectively.

Based on the above analysis, $f(\cdot)$ is the unknown nonlinear function, which varies with the boundary conditions $B=\left[B_{1}, \ldots, B_{6}\right]$. Therefore, the exiting optimization algorithms cannot ensure that the RMDR be controlled within its required range.

\section{The Actual Control Status of Raw Meal Calcination Process}

The actual control state for raw meal calcination process is shown in Fig. 2. Operational engineer determine upper limits $y_{\max }$ and lower limits $y_{\min }$ of control loops setpoints by considering the maximum value $\beta_{\max }^{e}$ of content of $\mathrm{f}-\mathrm{CaO}$ in easy calcination stage, the raw calcination index $f_{c 1450}$, the maximum value $\beta_{\max }^{d}$ of content of $\mathrm{f}-\mathrm{CaO}$ in difficult calcination stage, boundary conditions $B$ (i.e., flow, ingredients and particle size) of raw meal, and offline measurement value $\gamma(T)$. Then the operator would adjust the setpoints of control loops using the boundary conditions $B$ of raw meal, offline measurement value $\gamma(T)$, and upper limits $y_{\max }$ and lower limits $y_{\min }$ of control loop setpoints. Since RMDR cannot be measured online continuously, the operators have to evaluate the RMDR through visual inspecting.

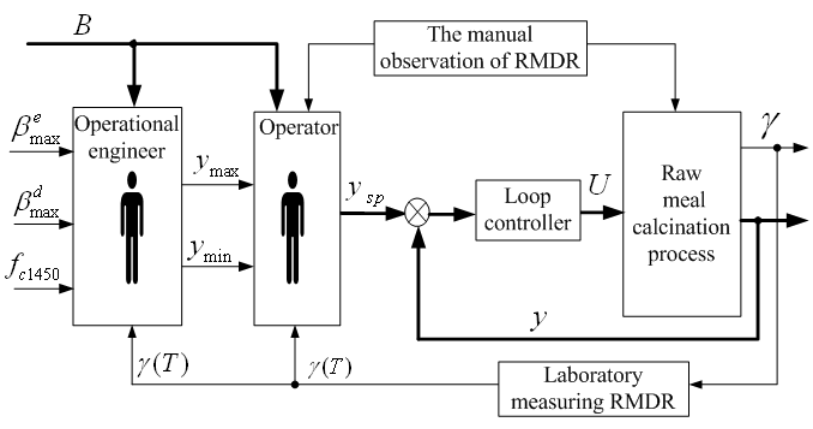

Fig. 2. The actual control state of raw meal calcination process

In fact, the operator cannot always obtain a correct setpoints $y_{s p}$ for the control loops by boundary conditions of raw meal. For example, when the iron oxide content $B_{1}$ in raw meal decreases, liquid phase would decrease, thus leading to the increase in RMDR.

The main task in this paper is to determine the RMDR target value $\gamma^{*}$ and the setpoints $y_{s p}$ of control loops so that the RMDR can be controlled within its required range and the production capacity is maximized.

\section{INTELLIGENT SETTING CONTROL METHOD FOR RAW MEAL CALCINATION PROCESS}

\section{A. Control Strategy}

To solve problem (2), an intelligent setting control method for raw meal calcination process has been proposed. The optimal control system is shown in Fig.3.

In Fig. $3, y=\left[y_{1}, y_{2}\right]$, and $y_{1}$ and $y_{2}$ are the feedback value of the calciner temperature and the preheater $\mathrm{C} 1$ outlet temperature, respectively; the manipulated variables $u=\left[u_{1}, u_{2}\right]$, and $u_{1}$ is the coal feeder speed and $u_{2}$ is the 
high-temperature fan damper. $B$ is the boundary conditions of raw meal, $\gamma^{*}, \gamma_{\text {soft }}(t), \gamma$ and $\gamma_{a u x}$ are the target value of RMDR, soft measurement value of RMDR, actual value of RMDR and auxiliary variable set, respectively; $\tilde{y}_{s p}(t)$ and $y_{s p}$ represent pre-setting value and final setting value of control loop, respectively; $y_{F}(t)$ and $y_{B}(T)$ denote feedforward compensation value and feedback compensation value, respectively; $T(T=k t)$ is laboratory measuring time, $f_{c 1450}$ is raw calcination index, $\beta_{\max }^{e}$ and $\beta_{\max }^{d}$ are the maximum value of $\mathrm{f}-\mathrm{CaO}$ in easy calcination stage and the maximum value of $\mathrm{f}-\mathrm{CaO}$ in difficult calcination stage, respectively.

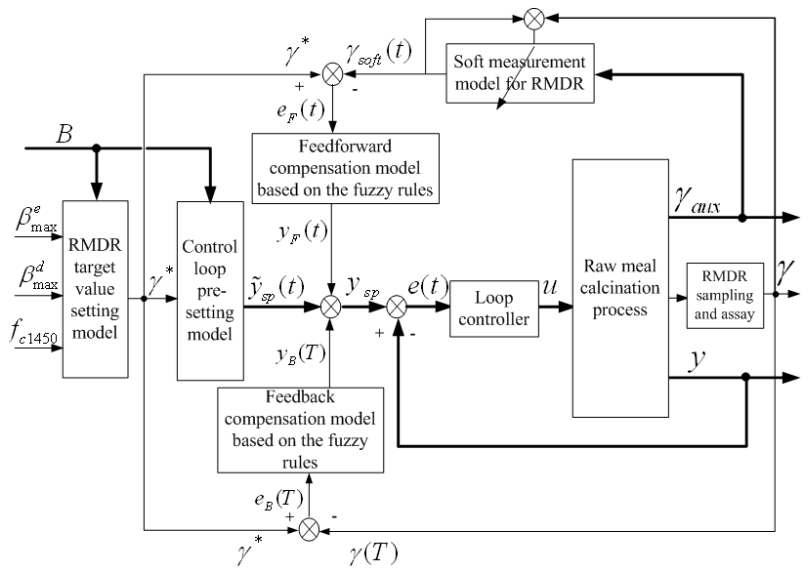

Fig. 3. The proposed intelligent setting control method for raw meal calcination process

In Fig.3, the intelligent setting control method for raw meal calcination process consists of a RMDR target value setting model, a control loop pre-setting model, a feedback compensation model based on the fuzzy rules, a feedforward compensation model based on the fuzzy rules, and a soft measurement model for RMDR. A detailed description for each module is discussed in section $\mathrm{B}$.

Thus, the final setpoint of control loops is given by:

$$
y_{s p}=\tilde{y}_{s p}(t)+y_{F}(t)+y_{B}(T)
$$

\section{B. Intelligent Setting Control Algorithm}

In order to realize (2), the intelligent optimization control algorithm is introduced in detail as follows.

1) RMDR Target Value Setting Model Based on SCM and $C$-ANFIS: In order to analyze relationship between the boundary conditions (i.e., $B_{1}$ and $B_{2}$ ) and the RMDR target value $\gamma^{*}$, the experiments were done when decomposition temperature is $870^{\circ} \mathrm{C}[7]$. The RMDR increased gradually when the content of $B_{1}$ or $B_{2}$ in raw meal increased. On the contrary, the RMDR decreased when the content of $B_{1}$ or $B_{2}$ in raw meal decrease. In addition, the RMDR target value is also different in easy calcination stage or difficult calcination stage. Based on the above analysis, the relationship between RMDR, $B_{1}$ and $B_{2}$ can be simple proportional relation. But change rate is unknown in different data scope. Thus, we adopt ANFIS to establish RMDR target value $\gamma^{*}$. Since the standard ANFIS cannot calculate the firing strength when the input contain numeric and categorical. At the same time, the model structure is difficult to determine. Based on the above analysis, a model based on SCM [8] and C-ANFIS was proposed to solve above problem, and its structure is shown in Fig. 4. For simplicity, input variable set $\bar{B}=\left[B_{1}, B_{2}\right]$ in RMDR target value setting model can be expressed as $x=\left[x_{1}, x_{2}\right]$.

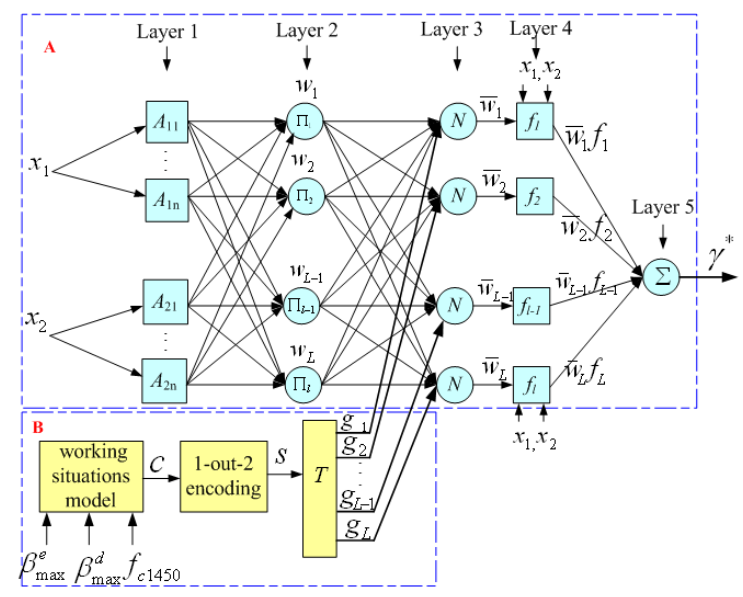

Fig. 4. RMDR target value setting model based on SCM and C-ANFIS

In Fig. $4, c$ is categorical input, $s$ represents twodimensional vector, $T$ denotes $2 \times L$ firing strength transform matrix, $g_{l}(l=1, \cdots, L)$ is the firing strengths of the $l$ th rule. Input variables of C-ANFIS are both numeric and categorical. The role of each function block in Part B and the node functions in Part A are described as follows:

The raw meal working situations model has the following features: If $f_{c 1450} \leq \beta_{\max }^{e}$, then categorical input $c$ represents easily calcination; If $\beta_{\max }^{e}<f_{c 1450} \leq \beta_{\max }^{d}$, then categorical input $c$ represents difficult calcination.

In Fig. 4, the categorical input $c$ is converted into twodimensional vector $s$ by 1-out-2 encoding. Thus the value of the two-dimensional vector $s$ are [1 0 ] and [0 1]. In addition, we can transform $s$ into the firing strengths $g_{l}(l=1, \cdots, L)$ using firing strength transform matrix $T$. Defining $G$ is a $L$ dimensional vector (i.e., $\left.\left[g_{1}, \ldots, g_{L}\right]\right)$, and $G$ can be expressed as

$$
G=s T
$$

Layer 1: Each node in this layer is square node and has the following node function

$$
O_{h}^{1}=\mu_{A_{h k}}\left(x_{h}\right), h=1,2, k=1, \cdots, n .
$$

Generally speaking, we choose $\mu_{A_{h k}}\left(x_{h}\right)$ to be bellshaped membership function, i.e.,

$$
\begin{gathered}
\mu_{A_{h k}}\left(x_{h}\right)=1 /\left(1+\left[\left(\frac{x_{h}-c_{h k}}{a_{h k}}\right)^{2}\right]^{b_{h k}}\right), \\
(h=1,2, k=1, \cdots, n),
\end{gathered}
$$

where $\left\{a_{h k}, b_{h k}, c_{h k}\right\}, h=1,2, k=1, \cdots, n$ are referred to as premise parameters $P^{p}$.

Parameter $c_{h k}$ in (6) is the center of the membership function $\mu_{A_{h k}}\left(x_{h}\right)$, and $c_{h k}$ is determined through SCM.

Layer 2: Each node in this layer is circle node labelled П. AND operator between fuzzy sets in this layer was 
performed to produce the firing strength $w_{l}$ of the lth fuzzy rule. The firing strength $w_{l}$ can be written as

$$
w_{l}=\prod_{i}^{2} \mu_{A_{i k}}^{l}\left(x_{i}\right), \quad(k=1,2, \cdots, n, l=1, \cdots, L) .
$$

Layer 3: Each node in this layer is circle node labelled $N$. All the rules' firing strengths in Part A were normalized. The node function in this layer is defined as $\bar{w}_{l}=$ $w_{l} g_{l} / \sum_{l=1}^{L} w_{l}, \quad(l=1, \cdots, L)$, where the firing strengths $g_{l}$ of the $l$ th rule can be calculated according to (4), and be expressed as $g_{l}=s \cdot T(l)$. The parameters in $T(l)$ is defined as $P^{m}$

Layer 4: Each node $l$ in this layer is square node with a linear function

$$
\bar{w}_{l} f_{l}=\bar{w}_{l}\left(p_{0}^{l}+p_{1}^{l} x_{1}+p_{2}^{l} x_{2}\right), \quad l=1, \cdots, L,
$$

where $f_{l}$ is the final output of the $i$ th fuzzy rule, $\left\{p_{0}^{l}, p_{1}^{l}, p_{2}^{l}\right\}, \quad l=1, \cdots, L$ are the consequent parameters $P^{c}$.

Layer 5: Node in this layer is circle node, and node function has the form

$$
\gamma^{*}=\sum_{l=1}^{L} \bar{w}_{l} f_{l}=\sum_{l=1}^{L} w_{l} g_{l} f_{l} / \sum_{l=1}^{L} w_{l} .
$$

Based on the above model, parameter identification and the structure of the C-ANFIS are fulfilled in the same manner as in [9].

2) Algorithm for Control Loop Pre-setting Model: Control loop pre-setting model imitate actions of excellent operators by input information, i.e. RMDR target value $\gamma^{*}$ boundary conditions $B$ of raw meal, and generate on-line pre-setting points $\tilde{y}_{s p}(t)$ of control loops when the raw meal boundary conditions changed. This pre-setting points $\tilde{y}_{s p}(t)$ can be expressed as:

$$
\tilde{y}_{s p}(t)=\left(\tilde{y}_{1 s p}(t), \tilde{y}_{2 s p}(t)\right)=\phi\left(\gamma^{*}, B_{1}, \ldots, B_{6}\right)
$$

where $\phi(\cdot)$ is an unknown nonlinear function varying with the raw meal boundary conditions $B$, thus existing algorithms can not solve (10) nonlinear mapping function. The case-base reasoning (CBR) [10] is generally suitable to the cases where precise mathematical model is not available. It uses experts' empirical knowledge to solve control loop pre-setting points. The CBR-based control loop pre-setting model for raw meal calcination is shown in Fig.5. This model consists of five components: namely the Case production for control loop pre-setting, the Case retrieval and case matching, the Case reuse, the Case base, and the Case revision and case retention.

Case production: According to the operators' excellent experience in raw meal calcinations process, the initial cases at different operating points were established. Then the case description of control loop pre-setting points can be expressed as follows:

$$
C_{k}:\left\{\left(T_{k}, X_{k}\right) \rightarrow Y_{k}\right\},(k=1, \cdots, n),
$$

where $n$ is the number of stored cases, $C_{k}$ represents the $k$ th case in case base, $T_{k}$ is producing case of time, and
$X_{k}=\left(x_{k, 1}, \ldots, x_{k, 7}\right)$ represent conditions describing $C_{k}$ in case base. In this context, $x_{k, 1}$ is the RMDR target value $\gamma^{*}, x_{k, 2}, \ldots, x_{k, 7}$ are the boundary conditions of $B_{1}, B_{2}$, $B_{3}, B_{4}, B_{5}$, and $B_{6}$, respectively. In addition, $Y_{k}$ denotes the solution (i.e., $\left.y_{k, 1}(t), y_{k, 2}(t)\right)$ of $C_{k}$ in case base, where $y_{k, 1}(t)$ and $y_{k, 2}(t)$ are the control loop pre-setting points of calciner temperature and preheater $\mathrm{C} 1$ outlet temperature, respectively.

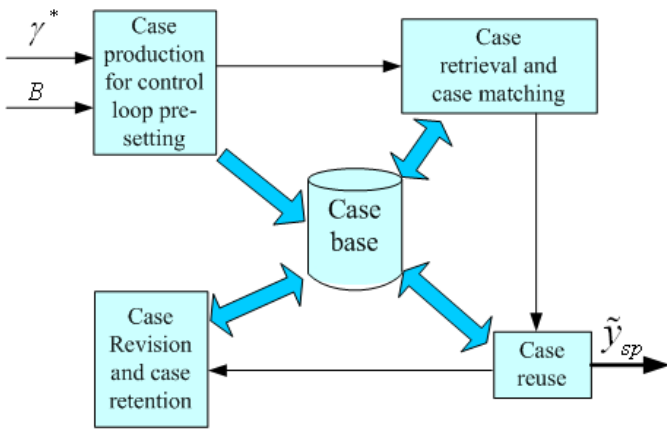

Fig. 5. CBR-based control loop pre-setting model for raw meal calcination process

Fifteen initial cases are obtained from the operational experience and these initial cases are stored in the Case base for case-based reasoning.

Case retrieval and case matching: Suppose that the current operating condition is $C_{c u r}$ and its description feature is $x_{i}(i=1, \cdots, 7)$. The cases $C_{k}$ in Case base possess the description feature of $x_{k, i}(i=1, \cdots, 7, k=1, \cdots, n)$ with $n$ being the number of stored cases. Thus, the similarity function $\operatorname{sim}\left(x_{i}, x_{k, i}\right)$ between the description feature $x_{i}$ of current operating condition $C_{c u r}$ and the description feature $x_{k, i}$ of the Case base is defined as

$$
\operatorname{sim}\left(x_{i}, x_{k, i}\right)=1-\left(\left|x_{i}-x_{k, i}\right| / \max \left(x_{i}-x_{k, i}\right)\right),
$$

where $k=1, \cdots, n, i=1, \cdots, 7$.

Thus, the similarity $S I M_{k}$ between the current operating condition $C_{c u r}$ and cases $C_{k}$ in Case base is given by

$$
\begin{gathered}
S I M_{k}=\sum_{i=1}^{7} \lambda_{i} \operatorname{sim}\left(x_{i}, x_{k, i}\right) / \sum_{i=1}^{7} \lambda_{i}, \\
(k=1, \cdots, n, i=1, \cdots, 7)
\end{gathered}
$$

where $\lambda_{i}$ denotes the weight coefficients of each case description feature obtained by expert's empirical knowledge. Similarity threshold $S I M_{y l}$ is defined as

$$
S I M_{y l}=\left\{\begin{array}{ll}
V, & \underset{k=1, \cdots, n}{\operatorname{Max}}\left(\operatorname{SIM}_{k} \geq V\right. \\
\underset{k=1, \cdots, n}{\operatorname{Max}}\left(S_{1}\right), & \underset{k=1, \cdots, n}{\operatorname{Max}}\left(\operatorname{SIM}_{k}\right)<V
\end{array},\right.
$$

where similarity threshold $V$ was determined by expert's experience.

All cases satisfying $S I M_{k} \geq S I M_{y l}$ were retrieved to act on the matching case.

Case reuse: Generally speaking, the situation does not exist in which the cases in Case base don't perfectly match the current conditions description feature. Detailed methods are as follows.

Suppose that we retrieve matching cases in Case base and it can be defined as $\left\{C_{1}, \ldots, C_{r}\right\}$. In addition, the similarity 
between the current operating condition $C_{c u r}$ and $C_{k}(k=$ $1, \cdots, r)$ is $S I M_{k}$ and corresponding case solution is $Y_{k}=$ $\left(y_{k, 1}, y_{k, 2}\right),(k=1, \cdots, r)$. Then, the case solution $\tilde{y}_{s p}$ for current operating condition $C_{c u r}$ is $\tilde{y}_{s p}=\left(\tilde{y}_{1 s p}, \tilde{y}_{2 s p}\right)$, where

$$
\tilde{y}_{l s p}=\left(\sum_{k=1}^{r} \lambda_{k} \times y_{k, l}\right) / \sum_{k=1}^{r} \lambda_{k},
$$

where $l=1,2$ and $w_{k}=S I M_{k},(k=1, \cdots, r)$.

Case revision and case retention: In this unit, $\tilde{y}_{s p}$ is adopted as new pre-setting points for control loop and case revision and case retention are fulfilled in the same manner as in [11].

3) Soft Measurement Model for RMDR: In practical production process, since RMDR $\gamma(t)$ cannot be measured online by a sensor, it can only be measured off-line once an hour. From (1), it can be seen that RMDR $\gamma(t)$ is a nonlinear function of the calciner temperature $y_{1}$, the preheater $\mathrm{C} 1$ outlet temperature $y_{2}$ and varying with the boundary conditions $B$. To sove this problem, a soft measurement model based on RFMPCA and LS-SVM is established in [12]. The auxiliary variable set of this model are listed in [12]. The RMDR value can be on-line calculated to obtain a feedforward compensation control.

4) Algorithm for Feedforward and Feedback Compensation: Although Pre-setting model based on CBR can give the pre-setting points of control loops according to the target value $\gamma^{*}$ and the boundary conditions $B$, the RMDR cannot still be guaranteed within its desirable ranges because of the fluctuation of the boundary conditions $B$. Since compensation value of control loop pre-setting points can't build a mechanism model, it can only be compensated by summing up excellent experts'experience. Therefore, the feedforward and the feedback compensators based on fuzzy rules are designed. Firstly, take for feedforward compensation model example, one thousand groups of input and output data were selected to extract fuzzy rules. Input data of fuzzy rules are the error $e_{F}(t)$ (i.e., $e_{F}(t)=\gamma^{*}-\gamma_{s o f t}(t)$ ) and the error of change $\Delta e_{F}(t)$, and $y_{F}(t)=\left[y_{F 1}(t), y_{F 2}(t)\right]$ as fuzzy rules output, where $y_{F 1}(t)$ and $y_{F 2}(t)$ denote the feedforward compensation value of the calciner temperature and the preheater $\mathrm{C} 1$ outlet temperature, respectively. The input and output compensation value were seen as input of subtraction clustering. Sample data set were written as follows:

$$
X^{1}=\left\{e_{F}(T), \Delta e_{F}(T), y_{F 1}, y_{F 2}\right\} \subset R^{4}
$$

The subtraction clustering algorithm, presented by Chiu [8], was used to determine clustering results of the rules numbers and membership functions center. Put the clustering center $x_{c i}(i=1, \cdots, n)$ of subtraction clustering algorithm as the center of the membership function. There are total five fuzzy rules after subtraction clustering. Take for $e_{F}(T)$ example, the center of the membership function as clustering center, therefore, five fuzzy set can be selected. We selected gaussian membership function as follows:

$$
\mu_{\gamma i}=\exp \left[-\left(e_{F}(T)-e_{c i}\right)^{2} / \sigma_{1 i}^{2}\right],(i=1, \cdots, 5),
$$

where $e_{c i}$ is error of the $i$ th clustering center. Similarly, we can determine fuzzy sets and membership functions of $\Delta e_{F}(T), y_{F 1}$ and $y_{F 1}$. The operation called Mamdani type inference was used in this condition. The center of gravity(COG) method [13] can be applied to the defuzzification to obtain the fuzzy controller output $y_{F i}(i=1,2)$.

To feedback compensation model, we use the same method as the feedforward compensation model to obtain the fuzzy controller output $y_{B j}(j=1,2)$.

\section{INDUSTRIAL APPLICATION}

The proposed intelligent setting control method has been successfully applied to the raw meal calcination process of Jiuganghongda Cement Plant in China. Fig. 6 shows the raw meal calcination process using short rotary kiln with suspended preheater and calciner. The rotary kiln is $48 \mathrm{~m}$ long, $3.2 \mathrm{~m}$ in diameter with 5 stage string preheater and a calciner. The control strategy proposed in this paper has been realized by using the software and hardware platform of the SIEMENS Series PCS7 and CEMAT.

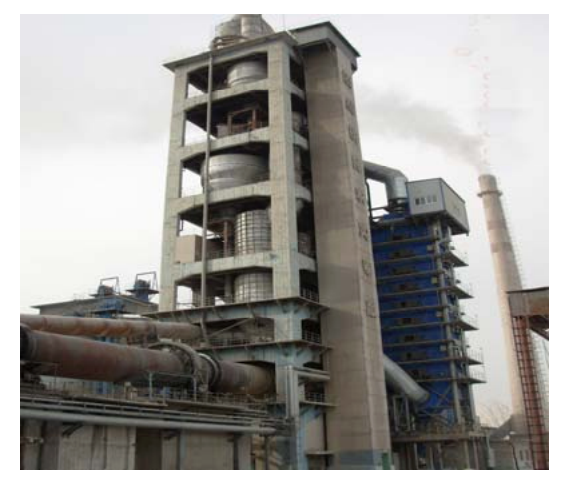

Fig. 6. Scene of raw meal calcination process in the plant

According to the production requirement, the maximum value $\beta_{\max }^{e}$ of content of $\mathrm{f}-\mathrm{CaO}$ in easy calcination stage is set to $\beta_{\max }^{e}=1.5 \%$ and the maximum value $\beta_{\max }^{d}$ of content of $\mathrm{f}-\mathrm{CaO}$ in difficult stage $\beta_{\max }^{d}=2.5 \%$. In the control loop pre-setting model, the weight values in (13) are defined as $\lambda=\left\{\lambda_{1}, \ldots, \lambda_{7}\right\}$ $=\{0.235,0.456,0.456,0.538,0.538,0.445,0.589\}$ and the threshold is 0.9 .

Application results of the proposed intelligent setting control method are shown in Figs.7-9. Figs.7-9 show the time varying trends of calciner temperature, $\mathrm{C} 1$ outlet temperature, and the manipulated variables (i.e., $\left(u_{1}, u_{2}\right)$ ) from 8:00AM to 4:00PM.

At 8:00AM, the raw meal is easy calcination since the raw calcination index $f_{c 1450}<\beta_{\max }^{e}$. Thus the RMDR target value $\gamma^{*}$ is set to 0.89 using (9). The control loop pre-setting model generated the pre-setting points $\tilde{y}_{s p}(8: 00)=\left[850^{\circ} \mathrm{C}, 330^{\circ} \mathrm{C}\right]$ of the control loops using (13) according to the RMDR target value $\gamma^{*}$, boundary conditions $B=\left[B_{1}, B_{2}, B_{3}, B_{4}, B_{5}, B_{6}\right]=$ $\left[2.32 \%, 13.72 \%, 3.6 \%, 17.4 \mu \mathrm{m}, 75 \mathrm{~T} / \mathrm{h}, 1010^{\circ} \mathrm{C}\right]$ of $\mathrm{raw}$ meal. In this case, the actual RMDR and the soft measurement values RMDR are both 0.88 . 
At 11:24AM, the raw meal is difficult calcination after 11:24AM since the raw calcination index $f_{c 1450}$ satisfy the following relationship: $\beta_{\max }^{e}<f_{c 1450}<\beta_{\max }^{d}$. At the same time, since $B_{2}$ declined from the original $2.32 \%$ to $2.01 \%$, therefore, the RMDR target value $\gamma^{*}$ is set to 0.93 , and control loop pre-setting model generated a new pre-setting points $\tilde{y}_{s p}(11: 24)=\left[880^{\circ} \mathrm{C}, 341^{\circ} \mathrm{C}\right]$ using (13).

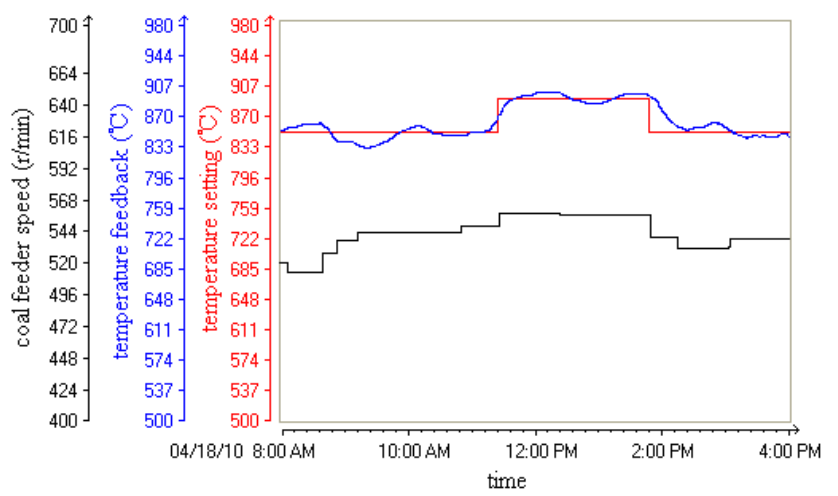

Fig. 7. The control curve of calciner temperature

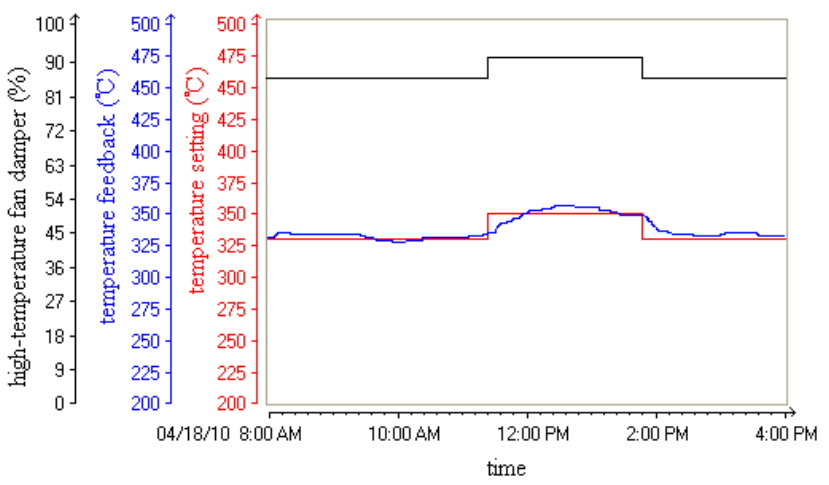

Fig. 8. The control curve of preheater $\mathrm{C} 1$ outlet temperature

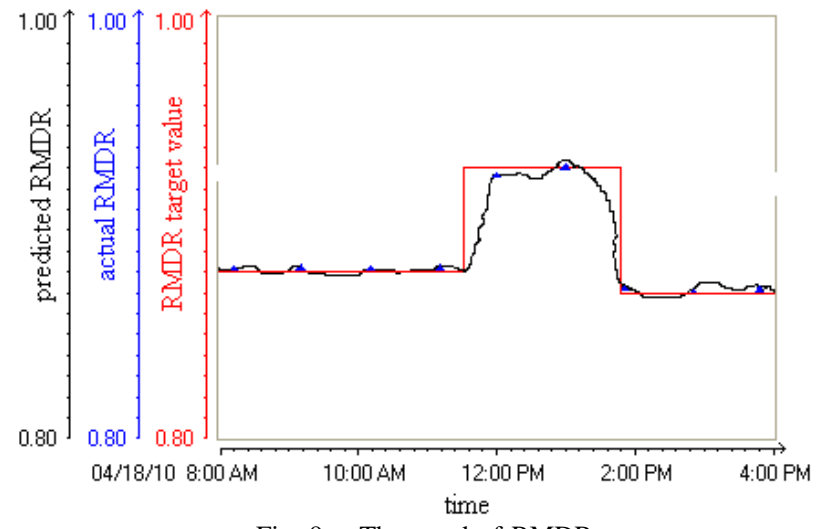

Fig. 9. The trend of RMDR

At 1:48PM, the raw meal is easy calcination since the raw calcination index $f_{c 1450}<\beta_{\max }^{e}$. In addition, since $B_{2}$ increased from the original $2.01 \%$ to $2.3 \%$ and $B_{5}$ declined from the original $17.4 \mu \mathrm{m}$ to $15.0 \mu \mathrm{m}$, thus the RMDR target value $\gamma^{*}$ is set to 0.85 using (9), and control loop pre-setting model generated a new pre-setting points $\tilde{y}_{s p}(1$ : $48)=\left[855^{\circ} \mathrm{C}, 335^{\circ} \mathrm{C}\right]$ using (13).

The SIEMENS control system was installed in April 2009. The raw meal calcination process was operated manually by operators before installation of the proposed strategy. After the control system was installed in April 2009, the user evaluated the system performance from April 2009 to August 2010 by RMDR, production capacity and equipment operation ratio. The sampling period for both was $1 \mathrm{~h}$. Using the proposed intelligent setting control method, RMDR is increased by 0.07 from previous 0.86 to the current 0.93 . However, equipment operation ratio has been increased by $2.55 \%$. Thus, production capacity is enhanced by $2.15 \mathrm{~T} / \mathrm{h}$ from previous $43.68 \mathrm{~T} / \mathrm{h}$ to the current $45.83 \mathrm{~T} / \mathrm{h}$.

\section{CONCLUSIONS}

This paper proposed an intelligent setting control method for raw meal calcination process to control the technology index, namely the decomposition ratio of raw meal (RMDR), with its desirable ranges according to the on-line adjusting the setpoints of the control loops with the change of raw meal boundary conditions. This method has been successfully applied to the raw meal calcination process of Jiuganghongda Cement Plant in China. Practical applications show that the proposed method has high potential in optimal operation and can be applied to the other complex industries.

\section{REFERENCES}

[1] K. P. Xie, NSP Cement Production of Fine Operation and Management, Beijing: Chemical industry press, 2007, Ch. 1.

[2] I. Makaremi, A. Fatehi, B. N. Araabi, and M. Azizi, "Abnormal condition detection in a cement rotary kiln with system identification methods," Journal of Process Control, vol. 19, pp.1538-1545, 2009.

[3] I. Iliuta, K. D. ,Johansen, and L. S. Jensen, "Mathematical modeling of an in-line low-NOx calciner," Chemical Engineering Science, vol. 57, pp. 805-820, 2002.

[4] [4] F. N. Koumboulis and N. D. Kouvakas, "Indirect adaptive neural control for precalcination in cement plants," Mathematics and Computers in Simulation, vol. 60, pp. 325-334, 2002.

[5] Y. Feng, Y. X. Chen, "Development of research on calcium carbonate for decomposed kinetics," Bulletin of the Chinese Ceramic Society vol. 25, pp. 140-146, 2006.

[6] Q. D. Chen,NSP cement technology principle and application, Beijing: China Building Materials Industry Press, 2004, Ch. 12.

[7] A. P. Li, W. Zhang, S. F. Jian, and D. H. Hu, "Studies on decomposition properties of cement raw meal in simulated precalciner," Journal of the Chinese ceramic society vol. 23, pp. 175-183, 1995.

[8] S. L. Chiu, "Fuzzy model identification based on cluster estimation," Journal of Intelligent and Fuzzy Systems, vol. 2, pp.267-278, 1994.

[9] M. Liu, M. Y. Dong and C. Wu, "A new ANFIS for parameter prediction with numeric and categorical inputs," IEEE Transactions on Automation Science and Engineering, vol. 7, pp. 645-653, 2010.

[10] N. Arshadi and I. Jurisica, "Data mining for case-based reasoning in high-dimensional biological domains," IEEE Transactions on Knowledge and Data Engineering, vol. 17, pp. 1127-1137, 2005.

[11] M. H. Tan and T. Y. Chai, "Modelling of the laminar cooling process with case-based reasoning," Control Theory and Applications, vol. 22, pp. 248-253, 2005.

[12] J. H. Qiao and T. Y. Chai, "Soft measurement model and its application in raw meal calcination process," Journal of Process Control, 2011 (Accepted).

[13] V. G. Moudgal, K. M. Passino and S. Yurkovich, "Rule-based control for a flexible-link robot," IEEE Transactions on Control Systems Technology, vol.2,pp.392-405, 1994. 